\title{
Dea del cielo o figlia di Eva? La donna nella cultura italiana fra Rinascimento e Controriforma, Museo petrarchesco piccolomineo, Trieste, 21 ottobre -21 aprile 2018.
}

Abstract

La mostra Dea del cielo o figlia di Eva? La donna nella cultura italiana fra Rinascimento $e$ Controriforma (Museo petrarchesco piccolomineo, Trieste, 21 ottobre - 21 aprile 2018), ha avuto il merito di congiungere il vasto patrimonio librario e della cultura materiale locale gettando nuova luce su aspetti inediti delle donne del tempo. Le varie sezioni della mostra e del relativo catalogo, curato da Maiko Favaro, evidenziano con efficacia le direttrici principali di questo percorso sul 'Rinascimento al femminile'.

\section{Recensione}

Nel 1561, presso i Guerra, il patrizio Giorgio Gradenigo allestisce un’importante antologia celebrativa per la poetessa e allieva di Tiziano, Irene di Spilimbergo, prematuramente scomparsa. La biografia che apre la raccolta, attribuita a Dionigi Atanagi, dopo un elenco delle 'tipiche' doti interiori, quali castità e ritrosia, nonché delle abilità pratiche consone allo status di gentildonna, quali il ricamo e il cucito, prosegue con il discorso sulla passione pittorica della giovane:

Et dando poco indugio al pensiero si pose al disegno [...] prendendo essempio solo dalle cose più perfette, come del Signor Titiano; [...] essendole fatto vedere un ritratto di Sofonisba Anguisciola, fatto di sua mano et rappresentato al Re Filippo, et sentendo meravigliose lodi di lei nell'arte della pittura, mossa da generosa emulatione, s'accese tutta d'un caldo desiderio di pareggiar quella nobile et valorosa donzella.

È una caratteristica nuova e rara, racchiusa nell'ossimoro della «virtuosa invidia», a costituire la cifra celebrativa della giovane, morta troppo presto per poter eccellere nella sua arte. Irene di Spilimbergo e il suo tombeau ben si prestano a evidenziare alcuni aspetti essenziali della mostra Dea del cielo o figlia di Eva?, ideata e curata da Maiko Favaro, con la collaborazione di Alessandra Sirugo, responsabile del Museo petrarchesco piccolomineo, che ha ospitato

The e-journal «altrelettere» is hosted at the URL: http://www.altrelettere.uzh.ch , in accordance with the Open Access Policy of the University of Zurich. Please cite this article as follows: Cristina ACUCCELLA, Dea del cielo o figlia di Eva? La donna nella cultura italiana fra Rinascimento e Controriforma, Museo petrarchesco piccolomineo, Trieste, 21 ottobre 21 aprile 2018 in «altrelettere», 3.08.2018, DOI: 10.5903/al_uzh-41.

(C) This article is licensed under a Creative Commons Attribution 2.5. Switzerland (CC BY-NC-ND 2.5). Please read the license terms on the website: http://creativecommons.org/licenses/by-nc-nd/2.5/ch/deed.en 
l'esposizione. Venezia, capitale editoriale del tempo, risulta l'ultima tappa di un complesso gioco di incontri, contatti e scambi che rivelano un insolito dinamismo artistico e letterario del mondo femminile del tempo, cui proprio l'allestimento in questione, con la cospicua e preziosa presenza di cinquecentine, tutte provenienti dalla biblioteca Attilio Hortis, congiunta a numerosi oggetti di cultura artistica e materiale, attinti da importanti collezioni dei musei di Trieste, Gorizia e Udine, ha il merito di valorizzare e riunire in un articolato gioco di risonanze e richiami reciproci. Ecco dunque comparire, tra gli altri, il Ritratto di monaca attribuito alla sorella di Irene, Emilia di Spilimbergo, copia da Sofonisba Anguissola (scheda 8), l'artista che per il suo talento pare aver aperto la strada all'accettazione sociale delle donne pittrici.

Questo particolare cortocircuito è sintomatico di una rete ben più complessa e ampia di quanto si possa pensare, così come l'occasione espositiva ha consentito di indagare, osservando il mondo artistico e letterario femminile sia dall'interno (ovvero sul piano dell'auto-rappresentazione), sia dall'esterno, vale a dire mediante l'immagine che di esso ci è restituita e filtrata dalla cultura maschile, nell'arco di tempo che va dagli albori del Rinascimento alla Controriforma. Gli studi di genere incentrati sul ' 500 , resi attuali in anni relativamente recenti, e sempre più in voga, hanno potuto trovare in questa mostra, di cui è testimonianza anche il pregevole catalogo che ne è derivato, scritto da Maiko Favaro, con un'appendice di descrizioni bibliografiche di Alessandra Sirugo, una valida opportunità di arricchimento di prospettive, almeno altrettante quante le sezioni in cui si è articolato. La prima ha indagato gli scritti sull'eccellenza femminile, vero e proprio filone saggistico che, partito alla fine del " $400 \mathrm{si}$ afferma con più decisione all'inizio del '500, in cui molti uomini, spesso impegnati in una sorta di mecenatismo culturale a favore delle donne, si ingegnano per decantarne il valore intellettuale e artistico, negletto da una cultura dominante che fino ad allora aveva lasciato loro uno scarso spazio espressivo, nonché sul protagonismo culturale delle donne stesse, artefici in prima persona di opere che per valore concorrono, nelle corti e al di fuori di esse, con quelle degli scrittori più noti del tempo. 
La seconda sezione si focalizza sul tema del canto per la lontananza dell'amato, la cui origine coincide con quella della lirica amorosa occidentale, nel provenzale amor de lohn, la cui resa al femminile trova un importante modello classico delle Heroides virgiliane, i cui lamenti risuonano nell'eco delle loro 'emule' in volgare, quali la Fiammetta boccacciana e la Bradamante del Furioso ariostesco (schede 19-20-21). La complessità del femminile trova poi una sua collocazione nella sezione dedicata ai dialoghi, laddove, sulla scia della Diotima platonica, dell'apporto ficiniano al tema e dell'esempio del Decameron e del Filocolo, le donne, da una parte sono per la prima volta ammesse a pieno titolo a prendere parte a discussioni di vario genere, dall'altra vedono tuttavia il loro contributo partecipativo circoscritto a temi 'tipicamente' femminili, quali la bellezza e l'amore, come avviene negli Asolani di Bembo e nel Raverta di Betussi; in ogni caso, come insegna il Cortegiano di Castiglione, sarà sempre per loro molto più decoroso, in presenza di uomini, 'parlare poco' (schede 26-30).

La quarta e ultima sezione è quella che ha indagato la più netta polarità tra la figura femminile nella sua più alta e inafferrabile trasfigurazione, quella della Vergine, risultato della temperie controriformistica, e la sua antagonista, Eva, che pure conserva un fascino diabolico esemplarmente incarnato, tra le altre, nell'Angelica del Furioso e nell'Armida della Gerusalemme liberata.

Il percorso tra testi, arti figurative e oggetti della vita quotidiana apre un quadro complesso, sfaccettato, del mondo femminile del tempo, in cui lo spettatore può addentrarsi e tracciare facilmente un fil rouge lungo il secolo di storia letteraria e di costume qui sapientemente ritagliato. Le 'figure di carta', che la storia letteraria ricorda come voce importante e 'altra' rispetto a quella maschile, si rivelano ora in carne ed ossa, come notiamo dai bustini e dai corpetti testimoni delle diverse estrazioni sociali delle donne e delle bambine che li indossarono (schede 10-11); le scopriamo inoltre scienziate, con i loro corredi ad uso farmaceutico (scheda 14), ora musiciste, di cui sono prova i liuti, strumenti tra i più usati anche in accompagnamento alla composizione poetica (schede 1213). 
Anche in via trasversale rispetto alle varie sezioni in cui la mostra si è articolata, le possibilità di rintracciare richiami a distanza sono molteplici. Tra i ritratti, quello anonimo di Vittoria Colonna, modello e creatrice del logos del petrarchismo femminile, prima a cantare la lontananza nell'epistola in terzine Eccelso mio signor, questa te scrivo, la cui prima attestazione si ha nel compendio di vocaboli del toscano ad opera di Fabrizio Luna (scheda 18). Il Furioso, vero best seller del tempo, non solo è qui presente in quanto opera d'arte, nella pregiata edizione de' Franceschi, riccamente illustrata in ogni canto (scheda 21), ma anche come opera in cui la donna conserva una particolare ambivalenza. La ritroviamo come emblema di devozione incondizionata in Isabella, eroicamente fedele al suo Zerbino, come anche sottolinea l'esemplare incluso nella raccolta del Donato, che dedica all'episodio degli ultimi sospiri dell'amato il madrigale Zerbin la debil (scheda 22), ma anche come figura inafferrabile di Angelica, principale oggetto del desiderio dei cavalieri, causa della follia di Orlando, elemento al quale fa da perfetto contrappunto la 'vendetta' del Brusantini, nel cui seguito dell'opera, la principessa del Catai, come in un dantesco contrappasso per analogia, si innamora di chiunque incontri (scheda 24). A un secondo livello, il Furioso non manca neppure di essere oggetto della 'critica' femminile, di cui è particolare testimonianza il Discorso sopra il Principio di tutti $i$ canti d'Orlando Furioso della napoletana Laura Terracina (scheda 25).

Il 'lato oscuro' del femminile, la cui origine coincide biblicamente con quella del mondo, come ben raffigura la testa del serpente con sembianze femminili, quasi alter ego di Eva, nel dipinto attribuito a Goltzius, ispirato alla famosa incisione di Dürer (scheda 36), esercita notevole attrazione, di cui rimane emblema incontrastato la demoniaca e irresistibile seduzione dell' «empia Armida», causa, con le sue «opre e frodi» (Gerusalemme Liberata, XIV 50-51), della perdizione dell'esercito cristiano. La conversione della «rea maga» in «ancilla» del suo Rinaldo, nel momento decisivo della battaglia tra cristiani e pagani (canto $\mathrm{XX}$ ), pone in risalto la redenzione cristiana, mutuando nel dettato la professione di fede pronunciata da Maria al momento dell'Annunciazione, 
mentre, dal canto suo, la devozione mariana post-tridentina (schede 38-42) sembra risolvere, in una contemplazione estatica e liturgica, tutte le contraddizioni del femminile.

In un ventaglio necessariamente irriducibile alla manichea distinzione tra 'perdizione' e 'santità', è possibile che trovi spazio una zona intermedia, frutto della misericordia divina, di cui è peculiare incarnazione un'altra figura emblematica della Controriforma, Maria Maddalena (scheda 43). Prima che il Barocco riesca a riassorbire tali dicotomie in una rinnovata misoginia, la veneziana Tarabotti giunge perfino ad elencare, confutando le autorità del passato, la nobiltà del sesso femminile (scheda 35) parallelamente ai difetti del sesso maschile, dopo che, dal canto suo, il Malipiero, indotto da scrupoli sull'eccessiva vanitas di Francesco e la sua passione peccaminosa verso la 'creatura' più che verso il suo 'Creatore', si era sentito in dovere di riscrivere interamente in chiave religiosa la lirica d'amore del Canzoniere petrarchesco (scheda 37).

Se ancora nel '700 le rivendicazioni delle voci femminili troveranno un contrappeso emblematico nell'Angelica 'legata', con cui il Magni depotenzia, immobilizzandola, l'inaccettabile sfuggevolezza del personaggio (scheda 23), va individuato proprio nel Rinascimento, anche attraverso il sistema delle corti, il momento in cui le donne «fanno gruppo», secondo la celebre espressione di Dionisotti (1967: 237), sia come parti attive di un circuito fino ad allora precluso, sia, ancora, come idoli, anche intellettuali, emblematicamente collocati nel 'Tempio' (scheda 4), architettura 'editoriale' e insieme metafora di un discorso inneggiante alle più alte virtù, il cui rovescio è costituito dal divertissement di eloquenza dell'elogiatore galante. Dea del cielo o figlia di Eva? Alla fine del percorso l'interrogativo rimane aperto, e questo va senz'altro assunto tra i meriti più alti della mostra triestina, che ha posto dinanzi agli occhi del pubblico le possibili coordinate di un Rinascimento al femminile. 
C. ACUCELLA, Recensione a Dea del cielo o figlia di Eva? La donna nella cultura italiana fra Rinascimento e 6 Controriforma, Museo petrarchesco piccolomineo, Trieste, 21 ottobre - 21 aprile 2018, DOI: 10.5903/al_uzh-41.

\section{Bibliografia}

DionisotTi Carlo, Geografia e storia della letteratura italiana, Torino, 1967. 